\title{
Focal adhesion kinase regulates actin nucleation and neuronal filopodia formation during axonal growth
}

\author{
Mariola R. Chacón ${ }^{1, *}$, Ana I. Navarro ${ }^{1, \pm}$, German Cuesto ${ }^{2, \mp}$, Isabel del Pino ${ }^{1, \mp}$, Ricardo Scott ${ }^{1, \neq}$, \\ Miguel Morales ${ }^{2}$ and Beatriz Rico ${ }^{1, \S}$
}

\begin{abstract}
SUMMARY
The establishment of neural circuits depends on the ability of axonal growth cones to sense their surrounding environment en route to their target. To achieve this, a coordinated rearrangement of cytoskeleton in response to extracellular cues is essential. Although previous studies have identified different chemotropic and adhesion molecules that influence axonal development, the molecular mechanism by which these signals control the cytoskeleton remains poorly understood. Here, we show that in vivo conditional ablation of the focal adhesion kinase gene (Fak) from mouse hippocampal pyramidal cells impairs axon outgrowth and growth cone morphology during development, which leads to functional defects in neuronal connectivity. Time-lapse recordings and in vitro FRAP analysis indicate that filopodia motility is altered in growth cones lacking FAK, probably owing to deficient actin turnover. We reveal the intracellular pathway that underlies this process and describe how phosphorylation of the actin nucleation-promoting factor $\mathrm{N}$ WASP is required for FAK-dependent filopodia formation. Our study reveals a novel mechanism through which FAK controls filopodia formation and actin nucleation during axonal development.
\end{abstract}

KEY WORDS: FAK, Axon development, Hippocampus, Mouse

\section{INTRODUCTION}

The wiring of the nervous system relies on the ability of axons to locate and recognize their appropriate synaptic partners. To aid this process, developing axons are equipped with a highly motile and exquisitely sensitive structure at their tip: the growth cone (Dickson, 2002). Growth cones are enriched in both actin and microtubules, and they are organized in three main compartments: the distal peripheral region, the transitional region and the proximal central region. The peripheral region contains filopodia and lamellipodia, formed by two higher-order networks of actin filaments that are organized as linear bundles (filopodia) or in a diffuse mesh (lamellipodia) (Mattila and Lappalainen, 2008; Pak et al., 2008).

Filopodia are thought to be ultimately responsible for sensing the environment around the cell, as they contain the receptors for guidance molecules (Bechara et al., 2008; Nawabi et al., 2010; Ren et al., 2004; Steketee and Tosney, 2002). Activation of these receptors initiates signaling that promotes the reorganization of the cytoskeleton to trigger specific cellular responses (i.e. attraction, repulsion). This cytoskeletal reorganization includes the regulation of actin dynamics and, consequently, proteins that control the actin cytoskeleton are involved in different aspects of axonal

\footnotetext{
${ }^{1}$ Instituto de Neurociencias, Consejo Superior de Investigaciones Científicas and Universidad Miguel Hernández, 03550 Sant Joan d’Alacant, Alicante, Spain.

${ }^{2}$ Structural Synaptic Plasticity Laboratory, Center for Biomedical Research of La Rioja, CIBIR, 26006 Logroño, Spain.

*Present address: Max Planck Institute of Molecular Cell Biology and Genetics, 01307 Dresden, Germany

¥These authors contributed equally to this work

§Author for correspondence (brico@umh.es)

This is an Open Access article distributed under the terms of the Creative Commons Attribution Non-Commercial Share Alike License (http://creativecommons.org/licenses/by-nc-sa/3.0), which permits unrestricted non-commercial use, distribution and reproduction in any medium provided that the original work is properly cited and all further distributions of the work or adaptation are subject to the same Creative Commons License terms.
}

Accepted 7 June 2012 development. For example, promoters of actin filament elongation or nucleation, such as ARP2/3, ENA/VASP and N-WASP, are essential for neuritogenesis, axon growth, filopodia formation and branching (Dent et al., 2007; Dwivedy et al., 2007; Kwiatkowski et al., 2007; Shekarabi et al., 2005; Strasser et al., 2004; Suetsugu et al., 2002).

Relatively little is known about the molecular mechanisms that link the extracellular guidance signals to the dynamic reorganization of actin-based structures in the growth cone. One candidate to mediate such activities is focal adhesion kinase (FAK; PTK2 Mouse Genome Informatics), a large non-receptor tyrosine kinase protein that is highly enriched in the growth cones of neurons (Chacón et al., 2010; Contestabile et al., 2003; Menegon et al., 1999). FAK plays an important role in axon development (Rico et al., 2004; Robles and Gomez, 2006), and is required for axons to respond to different extracellular cues and adhesion molecules, such as semaphorin 3A (SEMA3A), netrin 1, ephrin A1 and L1 (L1CAM Mouse Genome Informatics) (Bechara et al., 2008; Chacón et al., 2010; Li et al., 2004; Liu et al., 2004; Ren et al., 2004; Woo et al., 2009). However, it remains unclear how FAK participates in these events. We previously showed that the actin cross-linking protein alpha-actinin lies downstream of FAK (Chacón et al., 2010), suggesting that FAK might directly relay extracellular information to the actin cytoskeleton during axon remodeling.

Here, we tested the hypothesis that FAK signaling regulates the actin cytoskeleton in growing axons. Using the Cre-loxP recombination system, we generated mice that lack FAK in hippocampal pyramidal cells. Our results demonstrate that filopodia dynamics are disturbed in growth cones lacking FAK, possibly owing to deficient actin turnover. We also identified the molecular mechanisms underlying this process by demonstrating that FAK mediates the phosphorylation of N-WASP (WASL - Mouse Genome Informatics), an actin nucleation-promoting factor that is known to regulate the number of filopodia at the growth cone. These results reveal a novel mechanism through which FAK can control filopodia formation and actin nucleation during axonal development. 


\section{MATERIALS AND METHODS}

Mice

We used a conditional Fak gene strain in which FAK could be deleted using Cre-loxP-mediated targeted recombination, as described elsewhere (Rico et al., 2004). Fak floxfllox (Beggs et al., 2003), NEX-Cre (Goebbels et al., 2006) and $\mathrm{NEX}$-Cre; Fak floxfllox were maintained on a CD1 background and crossed as described previously (Chacón et al., 2010). Animals were handled in accordance with the European Communities Council Directive of 24 November 1986 (86/609/EEC).

\section{Primary culture and transfection}

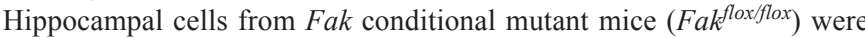
culture in poly-L-lysine $(0.5 \mathrm{mg} / \mathrm{ml})$ or poly-L-lysine and laminin $(5-10$ $\mu \mathrm{g} / \mathrm{ml}$ ) and transfected as previously described (Chacón et al., 2010). The plasmid $p C S 3-N-W A S P: G f p$ was generated by polymerase chain reaction (PCR) using as template a vector from (Miki et al., 1998) using the following primers: Fwd, 5'-CGGAATTCTCAGTCTTCCCATTCATCATCATCCTCAAAATC-3'; and Rev, 5'-CCCCCAAGCTTGGCCCGGGGATGAGCTCCGGCCAGCAGCAG-3'. The PCR product was subcloned into the $p E G f p-C 3$ vector (BD Bioscience). The $N$ WASPY256D:Gfp construct was obtained by PCR mutagenesis using $p C S 3-N-W A S P: G f p$ as the template plasmid and the following primers: Fwd, 5' -GAAACATCAAAAGTTATAGACGACTTCATTGAAAAAAC3'; and Rev, 5'-GTTTTTTCAATGAAGTCGTCTATAACTTTTGATGTTTC-3' (GenScript). Actin:Gfp constructs [PDGF-Actin:Gfp (Morales et al., 2000) or $p A c G F P: A c t i n$ (Invitrogen)] were co-transfected with Cre-EGfp or $p C D N A 3$ plasmids (0.5:1).

\section{Dil tracing and in utero electroporation}

To monitor the CA3-CA1 association projection, the lipophilic tracer 1,1' dioctadecyl-3,3,3',3'-tetramethylindocarbocyanine perchlorate (DiI) and GFP plasmids were placed or electroporated in the primordia of the CA3 region of the hippocampus from $\mathrm{Fak}$ mutant mice (NEX-Cre; Fakfloxflox $)$

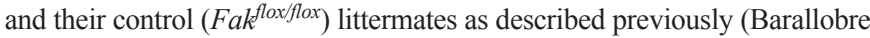
et al., 2000; Fazzari et al., 2010).

\section{Electrophysiology}

Coronal slices (200-300 $\mu \mathrm{m})$ were prepared as described previously (Price et al., 2008) and visualized on a SliceScope (Scientifica). Field potentials were acquired with an Axopatch 700B amplifier (Axon Instruments), filtered at $4 \mathrm{kHz}$ and digitized at $20 \mathrm{kHz}$. Stimulation of tracts was evoked by $40 \mu \mathrm{sec}, 90 \mathrm{~V}$ pulses through a bipolar electrode inserted $\sim 100 \mu \mathrm{m}$ deep into the axonal paths. Recordings were made blind to the genotype. Fiber volleys and field excitatory postsynaptic potentials (EPSPs) were recorded in CGP52432 $(5 \mu \mathrm{M})$, gabazine $(10 \mu \mathrm{M})$, NBQX $(40 \mu \mathrm{M})$ and DL-APV $(50 \mu \mathrm{M})$ (all Tocris Bioscience) through $\sim 1-\mathrm{M} \Omega$ glass pipettes filled with extracellular solution (Schmitz et al., 2000). For field EPSPs tetradotoxin (TTX, $1 \mu \mathrm{M}$; Tocris Bioscience) was added to subtract stimulation artifacts. Traces show average of five sweeps.

\section{Immunoprecipitation, immunocytochemistry,}

\section{immunohistochemistry and phalloidin staining}

For immunoprecipitation and immunoblotting, cortices including hippocampi from control and $F a k$ mutant mice were processed as described previously (Chacón et al., 2010). To detect in vivo phosphorylation of NWASP, $0.5 \mathrm{mM}$ pervanadate (orthovanadate, Sigma) was used in the lysis buffer.

For immunocytochemistry (IC), neuronal cultures were processed as described previously (Chacón et al., 2010). Antibodies used were: rabbit anti-FAK (Millipore), anti-N-WASP (Cell Signaling), anti-phosphoY256N-WASP (Abcam), mouse anti-actin (Sigma), anti-GAPDH (Sigma), goat anti-N-WASP (Santa Cruz) and chicken anti-GFP (Aves Lab). For histology, mice were perfused and processed as described previously (Sánchez-Huertas and Rico, 2011).

Cells were fixed and stained with phalloidin-488 (Molecular Probes) as described previously (Challacombe et al., 1996; Dent and Kalil, 2001; Schliwa and van Blerkom, 1981). To quantify the distribution of actin filaments in the growth cones, the areas stained with phalloidin were classified using the circularity parameter in ImageJ software (http://rsb.info.nih.gov/ij/docs/menus/analyze.html).

\section{Analyses of the growing axons and the growth cone morphology} To quantify the distance of the electroporated axons to the midline (before and after crossing), we took similar rostro-caudal electroporation at the hippocampus, and chose the 20 axons that extended the further distance within the inferior half of the commissure. To analyze growth cone volume in vivo, confocal imaging of the cones was carried out and a solid surface that exactly matched the contours of the growth cone was constructed for each one using Imaris (Bitplane Scientific Software). Surface area and background subtraction were identical between samples. In vivo, a filopodium was included if it was of uniform width and longer than $2 \mu \mathrm{m}$. In vitro, filopodia were considered as thin protrusions of uniform diameter $(<0.5 \mu \mathrm{m})$ ranging in length from 1 to $15 \mu \mathrm{m}$.

\section{Growth cone dynamics and fluorescence recovery after photobleaching (FRAP) analysis}

To study growth cone dynamics, recordings of the same growth cone were made at 6-8 days in vitro (DIV) over 5 minutes (30-second intervals) as described previously (Chacón et al., 2010; Rico et al., 2004). To analyze actin:GFP dynamics in living cells, individual growth cones with clear actin:GFP expression were selected for FRAP analysis as described previously (Martinez de Lagran et al., 2012). Pre- and post-bleaching images were obtained by attenuating the laser power to $3-5 \%$ to avoid significant photobleaching. Optimal bleaching was attained by increasing excitation power to $100 \%$. Background and acquisition photobleaching were corrected for each time point and intensity curves were normalized to initial fluorescence values (Rabut and Ellenberg, 2004). Mobile fraction was calculated as $\mathrm{Mf}=\left(\mathrm{F}_{\text {end }}-\mathrm{F}_{\text {post }}\right) /\left(\mathrm{F}_{0}-\mathrm{F}_{\text {post }}\right)$ where $\mathrm{F}_{\text {end }}$ is the mean region of interest (ROI) intensity at the equilibration time (75-100 seconds after bleaching), $\mathrm{F}_{\text {post }}$ is the ROI intensity after photobleaching and $\mathrm{F}_{0}$ is the mean ROI intensity at time 0 . Curves were fitted with Graphpad Prism v.4.0 software (Graphpad Software), assuming a one-phase exponential equation.

\section{RESULTS \\ The hippocampal commissural projection is disrupted in the absence of FAK}

Previous in vitro studies showed that FAK controls different aspects of axonal development in cortical neurons (Chacón et al., 2010; Liu et al., 2004; Rico et al., 2004). In particular, axons lacking FAK extend more slowly than normal (Rico et al., 2004). To investigate the function of FAK in vivo, we generated pyramidal cell-specific conditional Fak mutants by crossing NEX-Cre mice (Goebbels et al., 2006) with mice carrying a conditional Fak allele [Fak floxfflox mice (Beggs et al., 2003; Chacón et al., 2010)]. In NEXCre; Fak ${ }^{\text {floxfflox }}$ mutant mice, deletion of FAK is restricted to the forebrain and it results in a strong decrease of FAK expression by embryonic day (E) 16.5, although FAK is still detectable until postnatal day (P) 0 (supplementary material Figs S1, S3). Consistent with previous studies (Beggs et al., 2003; Rico et al., 2004; Watanabe et al., 2008), we found that loss of Fak function in pyramidal cells did not alter the gross morphology of the cerebral cortex (data not shown). To test the role of FAK in axonal development, we focused on the hippocampal commissural projection. This tract is formed by axons of CA3 pyramidal cells, which reach the midline at $\sim$ E16. By E17-18, commissural axons begin to invade the contralateral hippocampus, although this projection is not fully formed until P5 (Supèr and Soriano, 1994). Using DiI tracing in NEX-Cre; Fak floxflox mutant mice and their control littermates, we observed that reduction of FAK in hippocampal pyramidal cells did not prevent axons from reaching the midline at E16.5 (Fig. 1A,B). However, some of these axons 


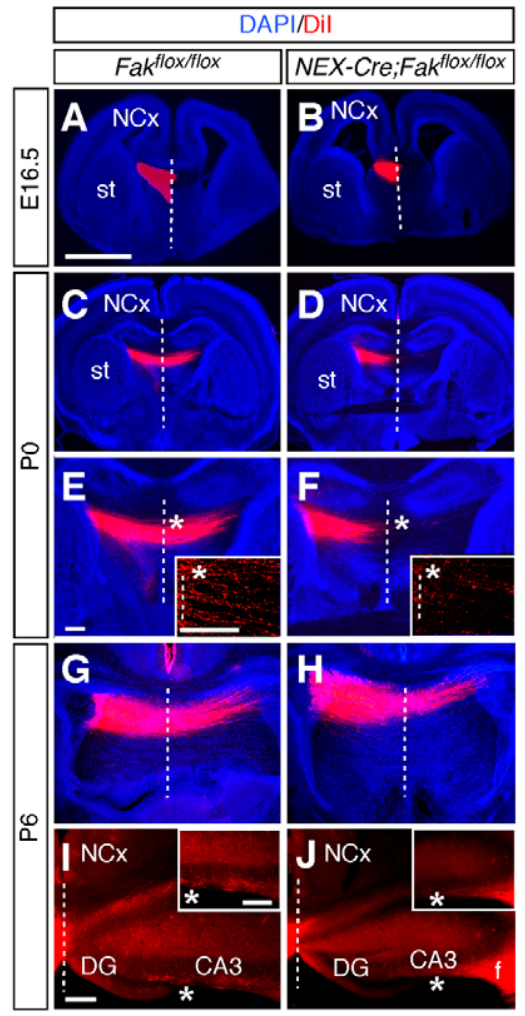

Fig. 1. Fak-deficient mice develop abnormal hippocampal commissural projections. (A-J) Fak flox/flox control mice $(A, C, E, G, I)$ and NEX-Cre; Fak flox/flox mutant mice $(B, D, F, H, J)$, after Dil injections into the hippocampus (E16.5: $n=5$ brains, two litters; P0: $n=8$ brains, three litters; P6: $n=6$ brains, two litters). (E,F) High magnification images from $C$ and D. (I,J) Dil-labeled axons in the contralateral hippocampus at P6. Asterisks show axons in the insets at higher magnification. Dashed line is the midline. DG, dentate gyrus; f, fimbria; NCx, neocortex; st, striatum. Scale bars: $1 \mathrm{~mm}$ for A-D; $500 \mu \mathrm{m}$ for E-J and associated insets.

failed to advance to the contralateral side at this stage (data not shown), a phenotype that was more evident at P0 (Fig. 1C-F). By P6, a moderate number of Fak-deficient hippocampal axons had crossed the midline (Fig. 1G-H), but did not innervate the contralateral hippocampus to the same extent as controls (Fig. 1I,J). To extend these observations and carry on quantitative analyses of growing axons, we transfected the enhanced green fluorescence protein (GFP) in control and Fak-deficient progenitor cells of the hippocampal primordium at E14.5 by in utero electroporation, and analyzed the distribution of axons at E18.5 and P0 (Fig. 2). Consistent with our previous experiments, we found that the average absolute distance to the midline for FAK-deficient axons was significantly smaller than for control axons (Fig. 2D,E,H-J). Indeed, a substantial percentage of FAK mutant axons were found close to the midline (Fig. 2K), failing to invade the contralateral hippocampus (Fig. 2L-O). These experiments reveal that axon outgrowth is delayed in vivo in the absence of FAK.

\section{Impaired function of the hippocampal commissure in Fak mutants}

To test the functional consequences of the axon outgrowth phenotype found in the previous set of experiments, we recorded electrical activity in the hippocampal commissure of FAK mutants and control mice at P0. Axonal firing was evoked by extracellular stimulation of one side of the ventral commissure. Fiber volleys (voltage deflections resulting from near simultaneous firing of bunches of axons) were recorded through an extracellular electrode inserted at decreasing distances from the stimulation site (Fig. 3A). The amplitude of $F a k$-deficient volleys significantly decreased with the distance from the stimulating electrode with respect to control volleys (Fig. 3B,C). These data are fully consistent with a delayed outgrowth of Fak-deficient axons in the hippocampal commissure (Figs 1,2), reinforcing the view that FAK is required for axonal growth in vivo.

Delayed axonal arrival at the contralateral hippocampus did not necessarily imply that overall neural transmission might be affected, because a compensatory increase in transmission could overcome the defective commissural pathway. To investigate the physiological consequences of FAK removal from hippocampal neurons, we recorded the synapses formed by commissural fibers contacting contralateral CA3 pyramidal cells in control and Fakdeficient mice at P6 (Fig. 3D). Bulk stimulation of the commissural path evoked postsynaptic responses recorded as field potentials (fEPSPs) in the CA3 pyramidal dendritic area. We found that the fEPSPs rising phase was significantly slower in Fak-deficient mice than in controls (Fig. 3E,F). These data indicate that the commissural path of Fak-deficient mice was less efficient at evoking postsynaptic responses than that of controls, probably owing to a decrease in the arrival of axons to the target area. In fact, consistent with the anatomical data (Figs 1, 2), fiber volleys of CA3 dendritic areas were smaller in Fak-deficient mice than in controls $\left(0.53 \pm 0.15 \mathrm{mV}, F_{k} k^{\text {flox/flox }}, n=7 ; 0.19 \pm 0.05 \mathrm{mV}, N E X\right.$ Cre; Fak floxfflox,$n=15 ; P<0.05$ by $t$-test). Our data demonstrated that impaired axonal outgrowth in Fak-deficient axons disrupts commissural function by reducing its capacity to stimulate CA3 pyramidal cells.

\section{Loss of FAK disrupts growth cone morphology in vivo}

As growth cones are largely responsible for axon growth and guidance, we wondered whether these structures were disrupted by the loss of FAK in hippocampal commissural axons. To investigate this, we analyzed the morphology of GFP-electroporated axons at E18.5, when both control and mutant axons are present at the hippocampal commissure. Using 3D reconstructions, we found that the average number of filopodia per growth cone is significantly smaller in Fak mutant axons than in controls (Fig. 4A-E). We also observed that the fraction of growth cones that had none or one filopodia represents $\sim 70 \%$ of the population of Fak-deficient growth cones. By contrast, $85 \%$ of control growth cones exhibited more than two filopodia (Fig. 4A-D,F). In addition, we found that the volume of Fak-deficient growth cones is significantly smaller than in control growth cones (Fig. 4G). Thus, the majority of the FAK mutant growth cones were found to be smaller than $25 \mu \mathrm{m}^{3}$, whereas the volume of control growth cones varied across a large range (Fig. 4H). These observations revealed that FAK is required for normal growth cone morphology in vivo.

\section{Growth cone dynamics are altered in Fak-deficient hippocampal axons}

We explored next whether the dynamic behavior of the growth cone was affected by the loss of FAK. To this end, we used realtime videomicroscopy to analyze in vitro the behavior of control and Fak-deficient growth cones. Consistent with our in vivo observations, we found prominent abnormal growth cone 


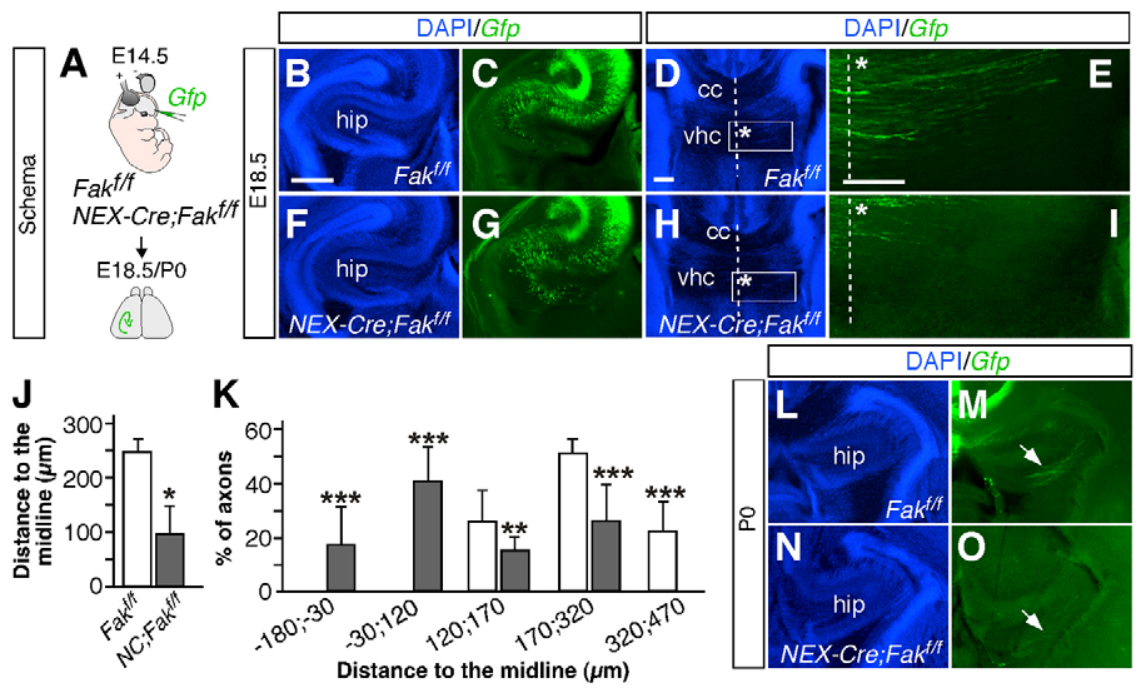

Fig. 2. Growing of commissural axons is delayed in the absence of FAK. (A-I) Experimental design is illustrated in A. Hippocampus and its commissure (E18.5) are shown from Fak ${ }^{\text {floxfflox }}$ (B-E) and NEX-Cre; Fak floxfflox (F-I) mouse embryos after in utero electroporation with a Gfp plasmid. E and I are high magnifications of boxed regions in $\mathrm{D}$ and $\mathrm{H}$, respectively. $(\mathbf{J}, \mathbf{K})$ Quantification of the axonal absolute distance to the midline $(\mathrm{J})$ and the relative distribution (percentage) of GFP-expressing axons at a given distance to the midline (K). Negative values show distance before the midline. $n=160$ axons, eight brains (four Fak flox/flox and four NEX-Cre; Fak floxfflox), four litters. ${ }^{*} P<0.05, t$-test $(\mathrm{J}) ; * * * P<0.0001 \chi^{2}$ test for entire distribution; ${ }^{*} P<0.001,{ }^{* \star *} P<0.0001, \chi^{2}$ test for individual distance distribution. (L-O) GFP-expressing axons (arrows) placed into the contralateral hippocampus to the electroporated site at PO ( $n=4$ brains, three litters). Dashed line is the midline brain. $c c$, corpus callosum; hip, hippocampus; NC, NEX-Cre; vhc, ventral hippocampal commissure. Error bars represent s.e.m. Scale bars: $200 \mu \mathrm{m}$.

morphology in FAK mutant growth cones. Time-lapse recordings with high temporal and spatial resolution revealed that the number of membrane events (extensions and retractions) was reduced in Fak-deficient growth cones compared with controls (Fig. 5A-C; supplementary material Movies 1, 2). Furthermore, whereas extension and retraction events were equally frequent in wild-type cones (extensions: 50\%; retractions: 50\%), Fak-deficient growth cones were more likely to extend than to retract (extensions: $72 \%$; retractions: $28 \%$; Fig. 5D,E). Thus, FAK is required for normal growth cone dynamics in vitro.
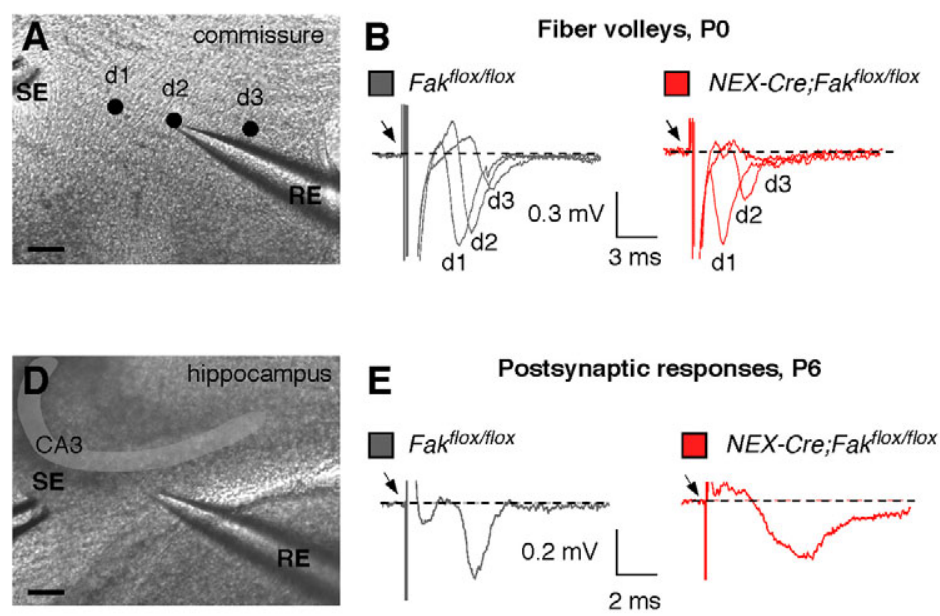
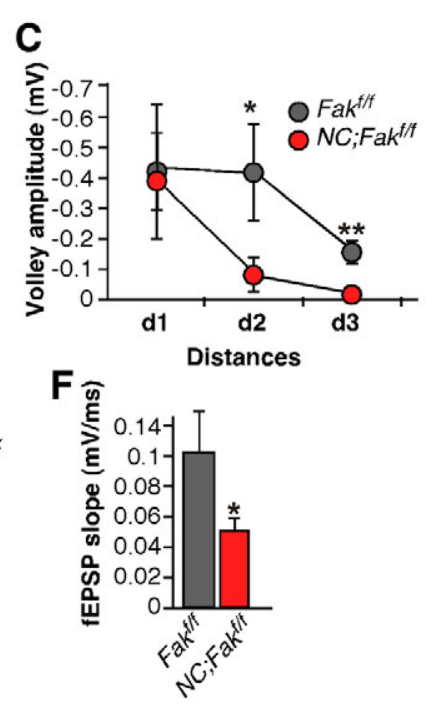

Fig. 3. Fak deletion reduces hippocampal commissure axonal firing and decreases the global transmission of commissural path to the contralateral hippocampus. (A-C) Bulk axonal firing in the hippocampal commissure at PO. (A) Hippocampal commissure (PO) showing a stimulating (SE) and a recording electrode (RE). Black dots indicate the recording sites at different distances from the stimulating electrode along the axonal tract direction (d1, d2 and d3). (B) Representative fiber volley traces. (C) Average amplitudes for Fak ${ }^{\text {flox/flox }}$ and NEX-Cre; Fak $k^{f l o x} / f$ lox fiber volleys along the hippocampal commissure ( $n=6$ Fak floxfflox and 7 NEX-Cre; Fak floxfllox ${ }^{*} P<0.01,{ }^{*} * P<0.001, t$-test). (D-F) fEPSPS at the CA3 pyramidal dendritic area (P6). (D) Image of the associational-commissural path contacting pyramidal CA3 layer. The SE is visible SE inserted at the entrance of the fiber path into the CA3 layer. The RE was inserted in the dendritic CA3 area. (E) Representative fEPSP traces. (F) Average of the rising slope of postsynaptic responses ( $n=7$ Fak floxfflox and 13 NEX-Cre;Fak flox/flox ${ }^{*} P<0.05, t$-test). NC, NEX-Cre. The black arrows indicate the onset of the stimulating artifact. Error bars represent s.e.m. Scale bars: $100 \mu \mathrm{m}$. 

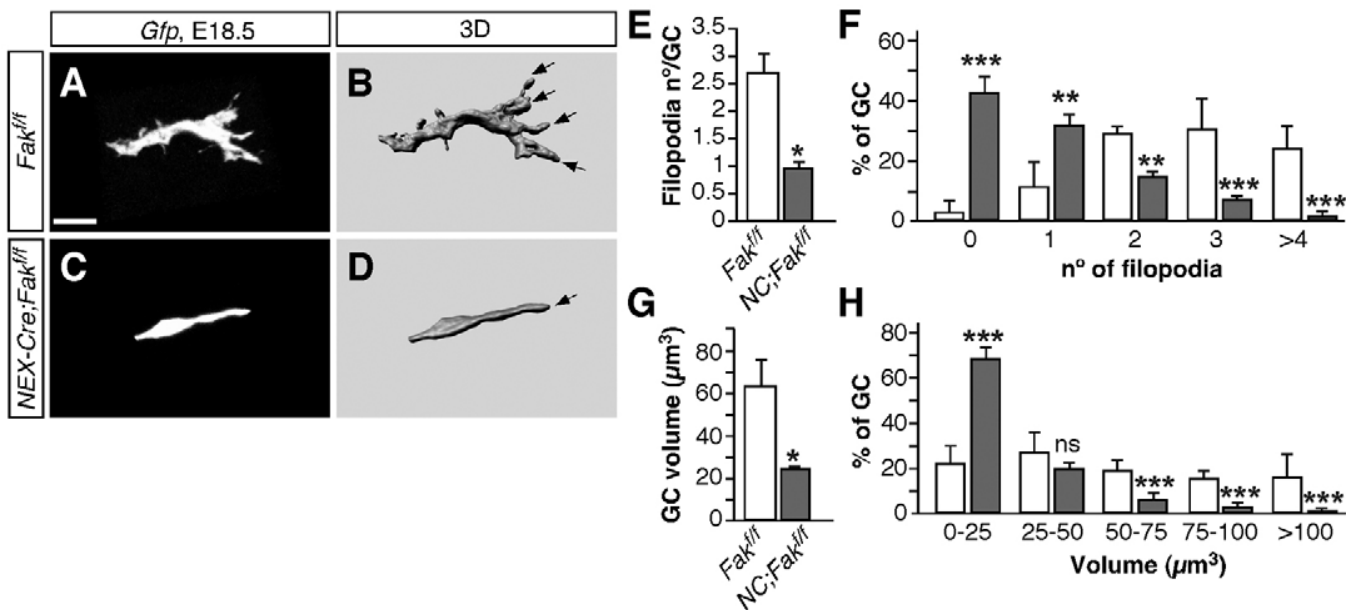

Fig. 4. Numbers of filopodia and growth cone volume are reduced in absence of FAK in vivo. (A-D) Control $(A, B)$ and $F A K-d e f i c i e n t(C, D)$ growth cones within the commissure (E18.5). (A,C) Confocal images. (B,D) 3D-Imaris reconstruction of $A(B)$ and C (D). (E,G) Quantification of filopodia number and volume per growth cone. $(\mathbf{F}, \mathbf{H})$ Quantification of the relative distribution (percentage) of growth cones filopodia number and

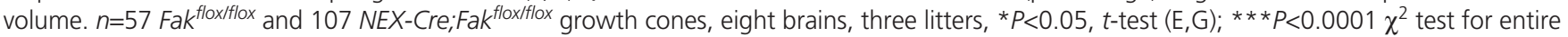
distribution $(\mathrm{F}, \mathrm{H}) ;{ }^{*} P<0.001,{ }^{*} *{ }^{*} P<0.0001, \chi^{2}$ test for individual distribution. GC, growth cone; NC, NEX-Cre. Black arrows show filopodia structures. Error bars indicate s.e.m. Scale bar: $5 \mu \mathrm{m}$.

\section{Abnormal organization of the actin cytoskeleton in the absence of FAK}

We observed that both the number of filopodia per growth cone and the growth cone area were smaller in Fak-deficient hippocampal axons than in controls in vitro (Fig. 6A,B,E,F). This phenotype was consistent with an impaired ability of growth cones to extend membrane protrusions in the absence of FAK. As growth cone dynamics are highly influenced by the remodeling of actin filaments (F-actin) (for reviews, see Dent et al., 2007; Pak et al., 2008), we investigated next the distribution of F-actin in the growth cone using phalloidin staining. We found that F-actin levels were strongly reduced in the peripheral region of Fak-deficient growth cones compared with controls (Fig. 6A-D). Moreover, the organization of F-actin filaments present in Fak-deficient growth cones differed considerably from controls. We used an automatic imaging algorithm to measure the circularity of the population of phalloidin-stained structures, which allowed us to classify F-actin filaments as either elongated (range 0-0.5, including long filaments) or circular (range 0.5-1, constituting short filaments). Growth cones lacking FAK contained fewer elongated and more circular structures than did wild-type growth cones (Fig. 6A,B,G). We also noticed that the peripheral region of FAK-deficient growth cones was almost entirely devoid of elongated F-actin filaments (Fig. 6B), a finding that is consistent with the reduced number of filopodia present in these growth cones.

In non-neuronal cells, FAK is typically activated following integrin-dependent formation of focal adhesions (Schaller et al., 1992). In neurons, FAK can be activated through its interaction with different membrane proteins, such as laminin-integrin complexes (Gupton and Gertler, 2010) or L1 (Bechara et al., 2008). Of note, filopodia formation in culture relies on different signaling pathways depending on the coating substrate. To investigate whether filopodia formation in FAK-deficient growth cones was dependent on the substrate, we replicated our previous experiments using laminin as substrate for the hippocampal cultures. As in the case of poly-L-lysine substrates, Fak-deficient neurons plated on poly-L-lysine/laminin substrates contained a smaller number of

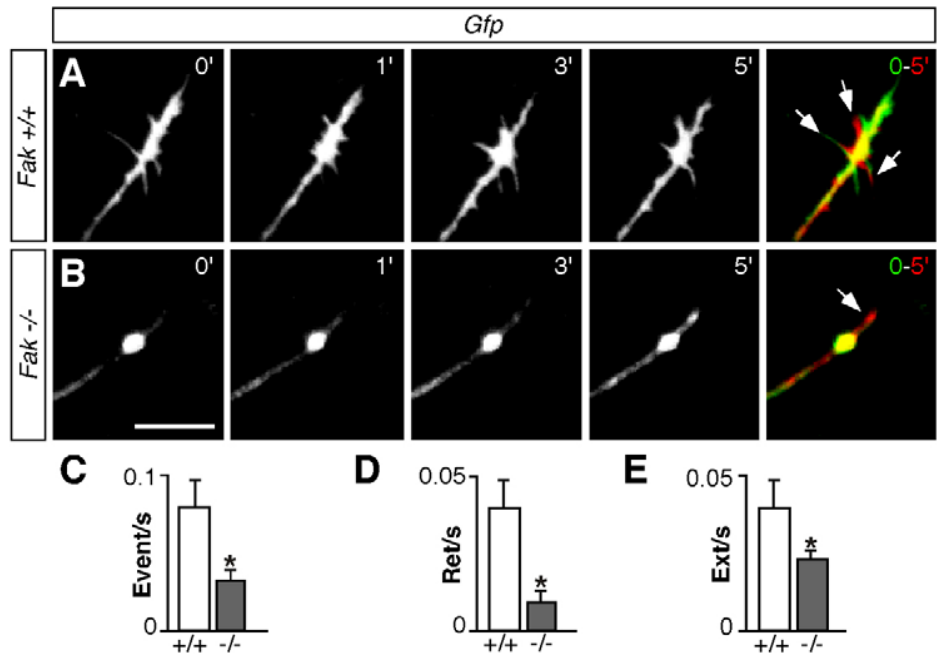

Fig. 5. Growth cones are less dynamic in the absence of FAK. (A,B) Video microscopy of the same growth cone from a Fak ${ }^{\text {floxfllox }}$ neuron transfected at 3 DIV with the Gfp reporter plasmid together with a mock $\left(\mathrm{A}, \mathrm{Fak}^{+/+}\right)$or $\mathrm{Cre}\left(\mathrm{B}, \mathrm{Fak}^{-/-}\right)$plasmid and recorded for 5 minutes at 6-8 DIV. The last panel shows superimposed images of the first $\left(t=0^{\prime}\right.$, green) and last $\left(t=5^{\prime}\right.$, red $)$ frames (extensions in red, retractions in green). White arrows indicate membrane events. (C-E) Quantification of total membrane events $(C)$, retractions (D) and extensions (E) per second $\left(n=38\right.$ growth cones, ${ }^{*} P<0.01, t$-test, where $15 \%$ of $\mathrm{Fak}^{+/+}$and $24 \%$ of $\mathrm{Fak}^{-/}$growth cones were paused and, therefore, excluded from the quantification). Error bars represent s.e.m. Scale bar: $10 \mu \mathrm{m}$. 


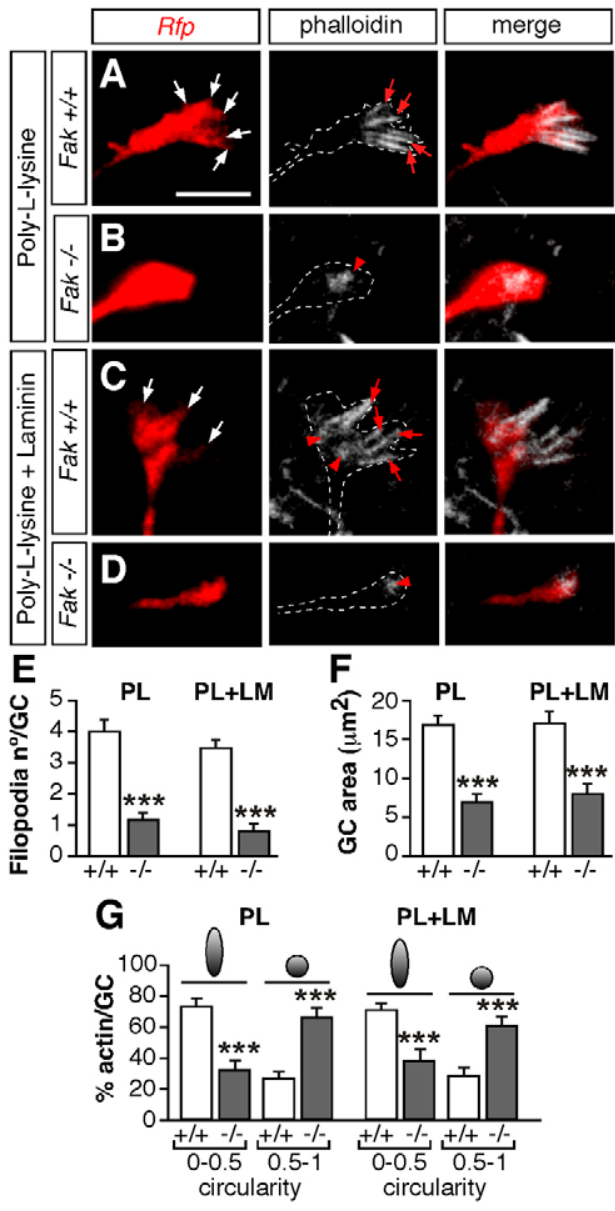

Fig. 6. Growth cone shape and actin cytoskeleton organization are impaired in FAK-deficient growth cones. (A-D) Growth cones from Fak floxfflox hippocampal neurons plated on poly-L-lysine $(A, B)$ or poly-L-lysine and laminin (C,D) transfected at 3 DIV with the $R f p$ reporter plasmid together with a control $\left(A, C, \mathrm{Fak}^{+/+}\right)$or $\mathrm{Cre}(\mathrm{B}, \mathrm{D}$, $\mathrm{FaK}^{-1-}$ ) plasmid and fixed at 6 DIV. Rfp-transfected growth cones were stained with phalloidin after fixation (white arrows, filopodia; red arrows, filamentous; red arrowheads, circular). Dashed lines outline the growth cones. (E,F) Quantification of filopodia number (E) and area (F) per growth cone. (G) Percentage of elongated and circular phalloidinstained structures per growth cone $(n=108$ growth cones from six independent experiments, $* * * P<0.0001, t$-test). Error bars indicate s.e.m. GC, growth cone; LM, laminin; PL, poly-L-lysine. Scale bar: $10 \mu \mathrm{m}$.

filopodia and growth cone area than controls (Fig. 6C-F). Likewise, FAK mutant growth cones displayed fewer elongated and more circular structures than wild-type growth cones (Fig. 6C,D,G). These results reinforce the view that the organization of actin filaments is altered in Fak-deficient growth cones, independently of the substrate used.

\section{Slow actin filament turnover in Fak-deficient growth cones}

We hypothesized that the loss of elongated F-actin structures, and hence of filopodia, in Fak-deficient growth cones could be due to deficient actin assembly into long filaments. To investigate actin dynamics in the growth cone, we performed fluorescence recovery after photobleaching (FRAP) experiments using Actin-Gfp. The total fluorescence recovery (mobile fraction) has two phases: a rapid initial phase (ms) and a slow component (s). It has been demonstrated that the slow phase, the major component of the mobile fraction, is mainly driven by the actin polymerizationdepolymerization turnover (Martinez de Lagran et al., 2012; Sprague and McNally, 2005; Star et al., 2002). We transfected control and Fak-deficient hippocampal neurons with a plasmid encoding Actin-Gfp. A $4 \times 4 \mu \mathrm{m}^{2}$ region at the leading edge of the growth cone was rapidly photobleached and the recovery of fluorescence in this region was monitored. As expected, our experiments revealed a fluorescence recovery profile with the two phases: the rapid component in the first 2-5 seconds that was probably due to the free diffusion of Actin-Gfp and the slow phase lasting 5-100 seconds that, as previously shown, mostly depends on actin polymerization (Star et al., 2002) (Fig. 7Q). We observed no differences between control and Fak-deficient growth cones in the kinetics of the rapid recovery and, likewise, it is identical to the free diffusion observed in neurons transfected with a $G f p$ expressing plasmid alone (data not shown). Together, these results indicate that the dynamics of free diffusion were not affected by the loss of FAK (Fig. 7R). Indeed, control growth cones tended to restore most of their initial fluorescence within 100 seconds (mobile fraction: $0.68 \pm 0.037$ ), indicating that the majority of actin filaments are being turned over continuously (Fig. 7A-D,Q,R). By contrast, the recovery of fluorescence in Fak-deficient growth cones over the same period of time was notably limited (mobile fraction: $0.35 \pm 0.041$; Fig. 7E-H,Q,R). Accordingly, the average mobile fraction of Fak-deficient growth cones decreased by $\sim 50 \%$ compared with controls, even though the initial fluorescence intensity was identical at the growth cone (data not shown). Similar results were obtained using poly-L-lysine/laminin substrates (Fig. 7I-P,Q,R), or disrupting FAK through the expression of FRNK (PTK2 - Mouse Genome Informatics), a dominant-negative form of FAK (Richardson and Parsons, 1996) (supplementary material Fig. S2). FAK might also modulate actin quantity at different levels (transcription and/or translation and/or degradation), as total actin protein was reduced in hippocampus lysates from Fak mutant mice (supplementary material Fig. S3). Nevertheless, overexpression of Actin-Gfp failed to rescue the filopodia-deficient phenotype of Fak mutant neurons $(n=269$ growth cones from three independent experiments, $P<0.001$ by ANOVA), suggesting that the deficits in filopodia formation found in FAK mutant neurons could not be directly explained by the reduction in actin levels. Altogether, these findings indicate that actin polymerization at the tips of the growth cone is perturbed in the absence of FAK.

\section{The activation and catalytic activity of FAK is required for filopodia formation and actin filament dynamics}

Having identified an influence of FAK activity on actin polymerization and filopodia formation, we investigated the possible mechanisms through which FAK might control this process. FAK and activated Src-family kinases (SFKs), recruited at FAK tyr397 upon FAK activation (Katz et al., 2002; Lietha et al., 2007; Owen et al., 1999; Schaller et al., 1999), have been proposed to phosphorylate the neural Wiskott-Aldrich syndrome protein $(\mathrm{N}$ WASP), an actin nucleation-promoting factor (Suetsugu et al., 2002; Wu et al., 2004). Thus, it is plausible that autophosphorylation of FAK, as well as the recruitment of SFKs, might regulate actin dynamics in the growth cone through $\mathrm{N}$ WASP. To test this hypothesis, we first performed rescue experiments by simultaneously transfecting $\mathrm{Fak} \mathrm{f}^{\text {floxfflox }}$ neurons with 


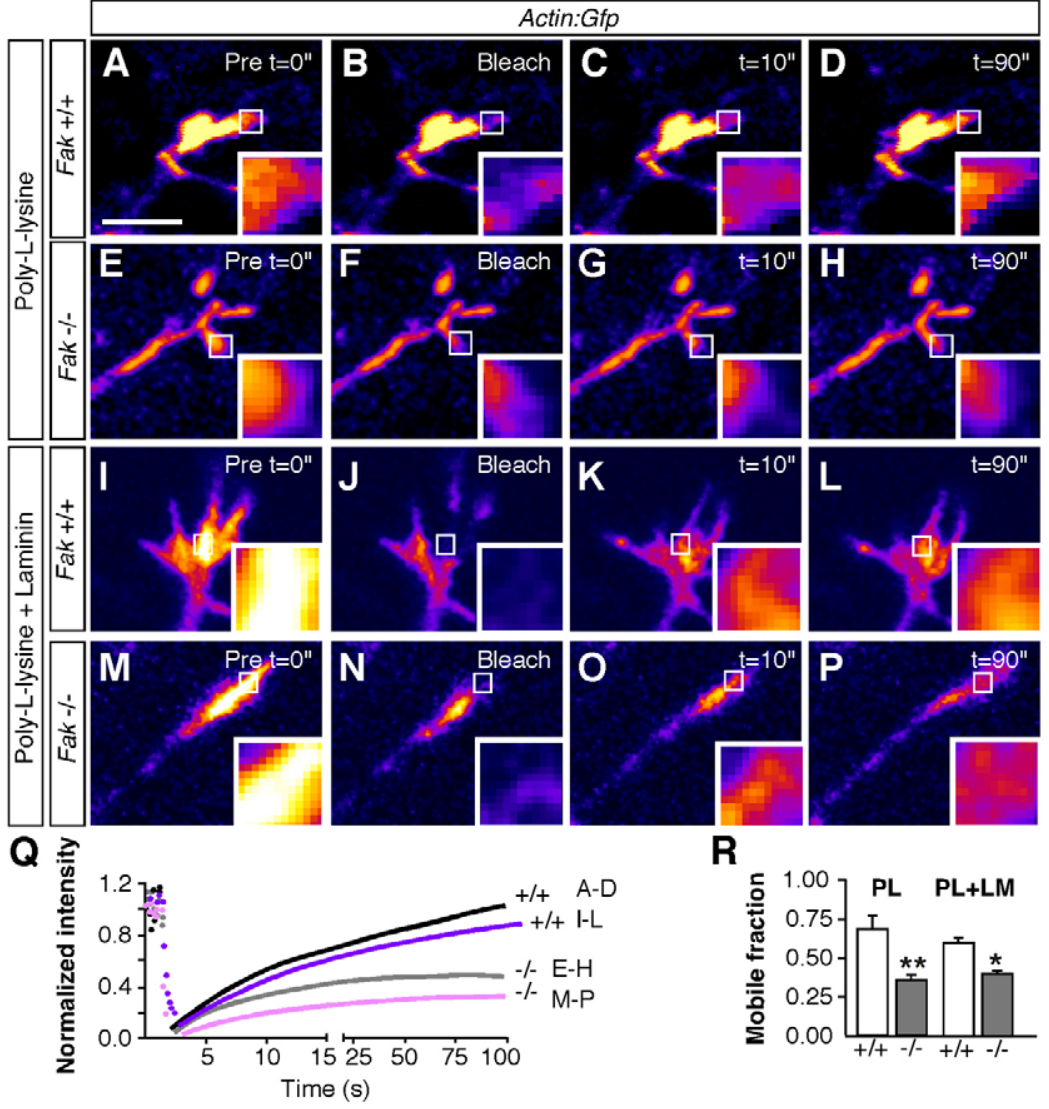

Fig. 7. FRAP reveals that actin filament turnover is reduced in Fak-deficient growth cones. (A-P) FRAP experiments on growth cones from Fak ${ }^{\text {flox/flox }}$

hippocampal neurons plated on poly-L-lysine $(\mathrm{A}-\mathrm{H})$ or poly-L-lysine/laminin (I-P) transfected at 3 DIV with Actin:Gfp together with control (A-D,I-L; $\left.\mathrm{Fak}^{+/+}\right)$or Cre $\left(\mathrm{E}-\mathrm{H}, \mathrm{M}-\mathrm{P} ; \mathrm{Fak}^{-1-}\right)$ plasmids and recorded at 6 DIV, at $\mathrm{t}=0$ (before photobleaching, Pre) and at $\mathrm{t}=0,10$ and 90 minutes after photobleaching. Boxed areas are shown at higher magnification. (Q) Fluorescence intensity profiles of the experiments illustrated in A-P showing the turnover of Actin:Gfp after 100 seconds (arbitrary units). (R) Quantification of the Actin:Gfp mobile fraction ( $n=150$ growth cones, eight independent cultures, ${ }^{*}{ }^{\star} P<0.001,{ }^{*} P<0.01, t$-test). Error bars represent s.e.m. LM, laminin; PL, poly-L-lysine. Scale bar: $10 \mu \mathrm{m}$ for A-P; $4 \mu \mathrm{m}$ for insets. plasmids encoding $\mathrm{Cre}$ and three different forms of FAK: wild-type FAK, a FAK isoform of containing a point mutation that disrupts Tyr397 phosphorylation $\left(F A K^{Y 397 F}\right)$, or a variant of FAK containing a point mutation that disrupts the kinase activity of FAK $\left(F A K^{K 454 R}\right.$, kinase dead). As expected, expression of wild-type Fak rescued the phenotype observed in Cre-transfected Fakflox/flox hippocampal growth cones (compare Fig. 6A,B and supplementary material Fig. $\mathrm{S} 3 \mathrm{~A})$. By contrast, neither $\mathrm{FAK}^{\mathrm{Y} 397 \mathrm{~F}}$ nor $\mathrm{FAK}^{\mathrm{K} 454 \mathrm{R}}$ rescued the number of filopodia or the normal arrangement of F-actin (supplementary material Fig. S4). These experiments revealed that the activation of FAK by autophosphorylation and its catalytic activity are both required for normal actin dynamics and filopodia formation in the growth cone.

\section{FAK-dependent $\mathbf{N}$-WASP phosphorylation is required for filopodia formation}

We studied next whether FAK was required for the function of $\mathrm{N}$ WASP in neurons. We observed no differences in the density of NWASP clusters in the segment of the axon closest to the growth cone (a section of axon at $10 \mu \mathrm{m}$ from the growth cone, $n=17$ axonal segments, three independent experiments, $P=0.58$ by $t$-test). By contrast, the density of N-WASP clusters was decreased in Fakdeficient growth cones compared with controls (Fig. 8A-I). In particular, there were fewer N-WASP clusters in the most peripheral regions of Fak-deficient growth cones than in controls (Fig. 8C,D,G,H). Loss of N-WASP was not due to a possible effect of FAK on N-WASP transcription, because overexpression of $\mathrm{N}$ WASP in Fak-deficient neurons failed to rescue the number of $\mathrm{N}$ WASP clusters (supplementary material Fig. S5), and total NWASP protein levels did not change in the absence of FAK (Fig. $8 \mathrm{~J}, \mathrm{~K})$. Interestingly, previous studies in NIH3T3 cells and macrophages have shown that phosphorylation of N-WASP/WASP influences its subcellular distribution (Cory et al., 2002; Wu et al., 2004), suggesting that FAK might regulate N-WASP localization in the growth cone of neurons through a similar mechanism. Consistently, we found that FAK formed native complexes with $\mathrm{N}$ WASP and phosphorylated N-WASP in vivo (Fig. 8J,K). Moreover, we observed that FAK was required for normal phosphorylation of N-WASP in cortical neurons (Fig. 8L,M). These results revealed that the subcellular distribution of N-WASP within the growth cone and its phosphorylation depend on FAK function.

In macrophages, expression of a constitutively phosphorylated form of WASP stimulates actin polymerization and induces extensive filopodia formation in vitro (Cory et al., 2002). To add further support to the hypothesis that abnormal N-WASP phosphorylation and distribution in Fak-deficient growth cones contributes to the defects in F-actin dynamics, we compared the distribution of wild-type N-WASP and its constitutively phosphorylated form $\left(N-W A S P^{Y 256 D}-G f p\right)$ in wild-type growth cones. We found that wild-type N-WASP was particularly abundant in the central domain of the growth cone, whereas $N-W A S P^{Y 256 D_{-}}$ $G f p$ accumulated preferentially in the peripheral domain of the growth cone compared with the central domain ( $N-W A S P-G f p$, supplementary material Fig. S6). This suggested that phosphorylation of N-WASP mobilizes this protein to the tips of the filopodia. We investigated next whether $N-W A S P^{Y 256 D}-G f p$ could restore filopodia formation and actin filament dynamics in the absence of FAK. We found that expression of $N-W A S P^{Y 256 D_{-}}$ $G f p$ in Fak-deficient neurons restores the number of filopodia to normal levels (Fig. 9A-E), a result that was not observed when neurons were transfected with wild-type N-WASP (Fig. 9A-E). In addition, the density and distribution of N-WASP clusters present 


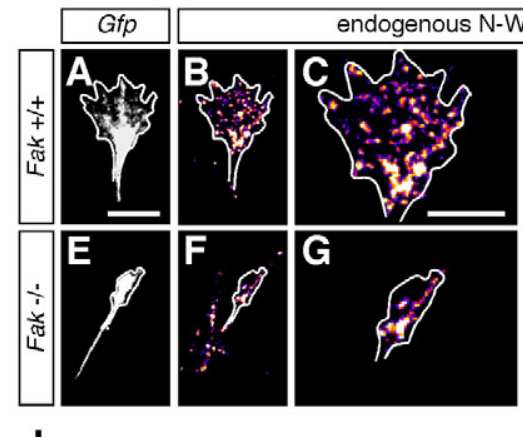

J

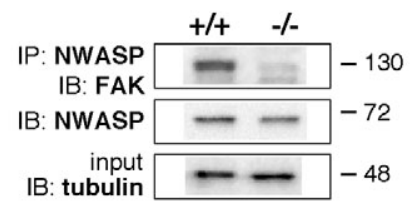

$\mathbf{L}$

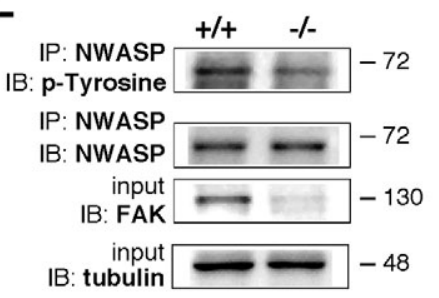

I

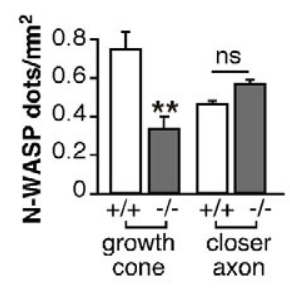

K

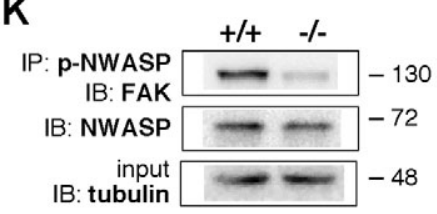

M

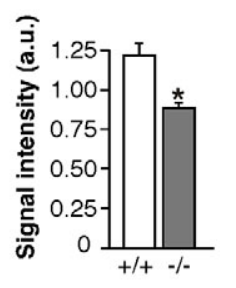

Fig. 8. N-WASP normal distribution in the growth cones requires FAK activity.

$(\mathbf{A}, \mathbf{E})$ Growth cones from Fak flox/flox hippocampal neurons transfected at 3 DIV with the $R f p$ reporter together with control $\left(\mathrm{A}, \mathrm{FaK}^{+/+}\right)$or $\mathrm{Cre}\left(\mathrm{E}, \mathrm{Fak}^{-/-}\right)$ plasmids and fixed at 6 DIV. (B,C,F,G) Pseudocolored images based on the fluorescence intensity in transfected growth cones of N-WASP immunostaining. (D,H) Binary images from $C$ and $G$, processed with ImageJ. (I) Quantification of the number of N-WASP positive puncta per $\mu \mathrm{m}^{2}$ of growth cone and $10 \mu \mathrm{m}$ length of the axon $(n=75$ growth cones, three independent experiments, ${ }^{*} * P<0.001, t$-test). (J,K) Co-immunoprecipitation of $\mathrm{N}-\operatorname{WASP}(n=6)$ and phosphorylated N-WASP $(n=5)$ with FAK from cortical homogenates, including hippocampus. (L) Tyrosine phosphorylation of $\mathrm{N}$ WASP. N-WASP was immunoprecipitated (IP) from cortical lysates, including hippocampus from Fak ${ }^{\text {flox } / \text { flox }}$ and NEX-Cre; Fak $k^{\text {floxfllox }}$ with anti-N-WASP antibody. (M) Quantification of N-WASP tyrosine phosphorylation by densitometry, $n=10$ controls and 12 mutants, three litters, ${ }^{*} P<0.05, t$-test. Error bars represent s.e.m. Scale bars: $5 \mu \mathrm{m}$. in Fak-deficient neurons transfected with the constitutively phosphorylated N-WASP were similar to those observed in controls (supplementary material Fig. S5). Finally, expression of $\mathrm{N}$ WASP $P^{Y 256 D}-G f p$ in Fak-deficient growth cones was also able to restore the relative percentage of actin elongated and circular structures to control levels (Fig. 9F). These results demonstrate that FAK promotes actin nucleation and filopodia formation in the growth cone through the direct or indirect phosphorylation of $\mathrm{N}$ WASP.

\section{DISCUSSION}

We have found that loss of FAK in hippocampal pyramidal neurons in vivo disrupts the normal morphology of growth cones, delays axonal outgrowth and disrupts the function of the associational commissure pathway. This phenotype is likely to be due to altered filopodia dynamics at the growth cone in the absence of FAK, as revealed by time-lapse recordings. Impaired filopodia formation correlates with deficient actin turnover in FAK-deficient growth cones, which appears to be the molecular basis for this phenotype. Indeed, we show that FAK controls actin nucleation by regulating the distribution of N-WASP. This process involves phosphorylation of N-WASP, which can rescue the defects in actin organization and filopodia formation found in FAK-deficient neurons. Our findings reveal a new mechanism through which FAK controls actin dynamics, promoting filopodia formation and axonal outgrowth during development.

\section{The role of FAK in axonal development: axon guidance and growth?}

Different functions have been identified for FAK during cortical axon development in vitro (Bechara et al., 2008; Chacón et al., 2010; Li et al., 2004; Liu et al., 2004; Ren et al., 2004; Rico et al., 2004). For example, several guidance cues require FAK to elicit appropriate responses in cortical axons, and both SEMA3A chemorepulsion and netrin 1 chemoattraction of cortical axons seems to require FAK, at least in vitro (Bechara et al., 2008; Chacón et al., 2010; Li et al., 2004; Liu et al., 2004; Ren et al., 2004). In addition, reduced FAK activity has been reported to impair midline crossing in Xenopus spinal commissural axons (Robles and Gomez, 2006). Similarly, netrin 1-deficient mice exhibit defects in axonal guidance across the midline in vivo (Barallobre et al., 2000; Serafini et al., 1996), but these abnormalities are far more severe than those reported here in FAK conditional mutants. Our genetic studies demonstrate that hippocampal axons do not require FAK to cross the midline. Instead, we found that FAK-deficient axons reach the contralateral hippocampus with some delay, linking FAK activity to axonal outgrowth in vivo. All the in vivo data is consistent with our previous observation that axons lacking FAK extend more slowly in vitro (Chacón et al., 2010; Rico et al., 2004) and suggests that FAK might not be essential in axons for responding to netrin 1 chemoattraction. Alternatively, undetectable traces of FAK might be still present in Fak mutant axons and support netrin 1 chemoattraction at the midline. Future investigations are needed to elucidate this. In any case, our experiments suggest that FAKdeficient axons have abnormal morphology and delayed axon growth.

Cells and growth cones move forward by coupling intracellular actomyosin-based motility to the extracellular substrate, or through contact with other cells via surface receptors (Suter and Forscher, 2000). There is strong evidence that FAK is required for the normal response of axons to different extracellular cues and adhesion molecules (Bechara et al., 2008; Chacón et al., 2010; Li et al., 2004; Liu et al., 2004; Ren et al., 2004; Woo et al., 2009), but a direct connection between FAK and the actin cytoskeleton, and its putative role in axonal development remained to be defined. Here, 


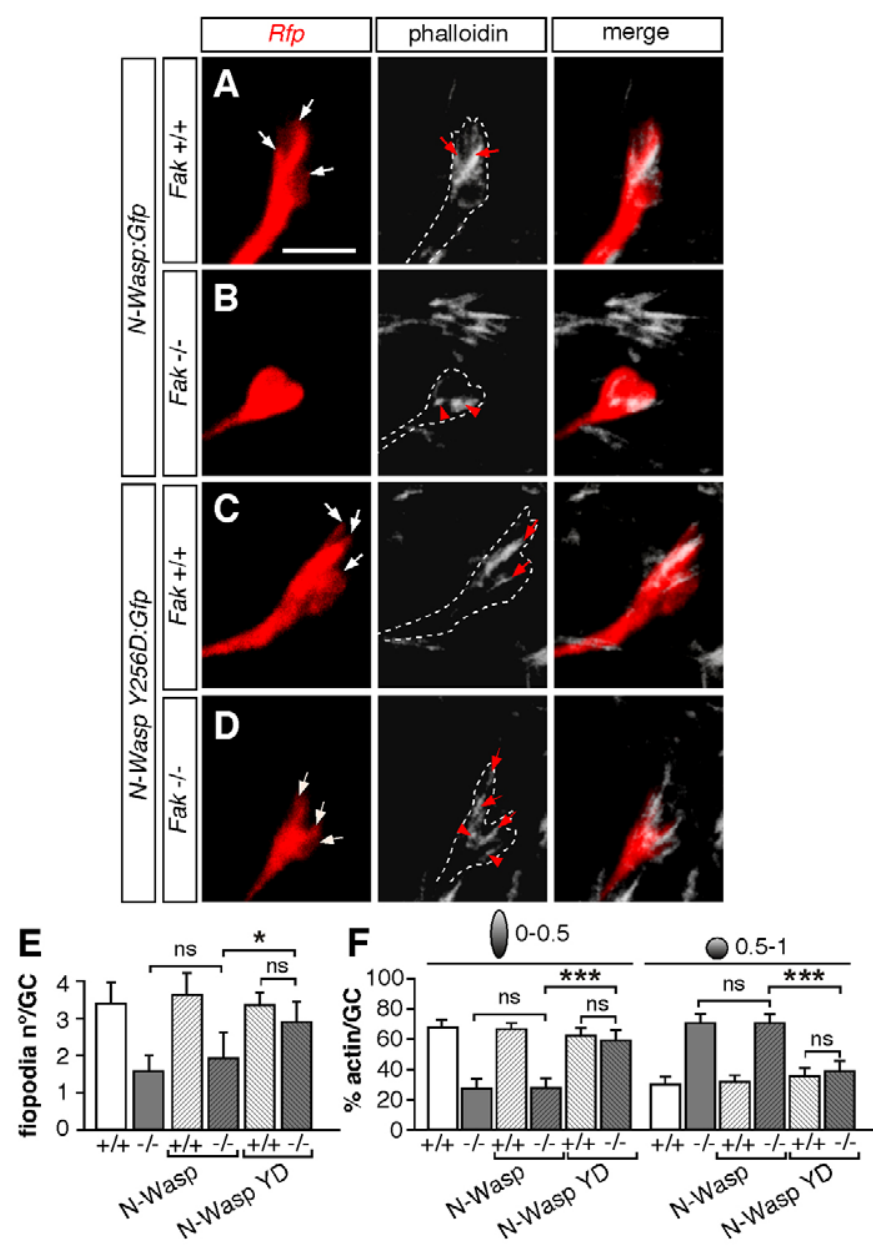

Fig. 9. N-WASP phosphorylation at Tyr256 is essential for FAKdependent filopodia formation and normal actin organization. (A-D) Growth cones from Fak ${ }^{\text {floxfflox }}$ hippocampal neurons transfected at 3 DIV with the Rfp reporter together with mock $\left(\mathrm{Fak}^{+/+}\right) \mathrm{N}$-WASPWT-Gfp (A), Cre (Fak $\left.{ }^{-1-}\right) N$-WASP ${ }^{W T}$-Gfp (B), mock + N-WASP ${ }^{Y 256 D}$-Gfp (C) and $\mathrm{Cre}+\mathrm{N}$-WASP ${ }^{Y 256 D_{-}} \mathrm{Gfp}(\mathrm{D})$ plasmids and fixed at 6 DIV. $R f p-$ transfected growth cones were stained with phalloidin after fixation (white arrows, filopodia; red arrows, filamentous; red arrowheads, circular). Dashed lines outline the growth cones. (E) Quantification of the number of filopodia per growth cone. (F) Percentage of elongated and circular phalloidin-stained structures per growth cone $(n=148$ growth cones, six independent cultures, ${ }^{*} * * P<0.0001,{ }^{\star} P<0.01$, ANOVA). GC, growth cone; LM, laminin; PL, poly-L-lysine; YD, $N$ WASP ${ }^{Y 256 D}$. Scale bar: $5 \mu \mathrm{m}$.

we have demonstrated that FAK mutant growth cones display altered actin dynamics that are caused, at least in part, by preventing N-WASP phosphorylation via FAK. Given that the disassembly, assembly and maturation of the adhesion contacts are all affected in the absence of FAK (Bechara et al., 2008; Chacón et al., 2010), it seems conceivable that uncoupling actin and point adhesions would alter growth cone motility in axons lacking FAK. Indeed, actin bundles appear to be randomly orientated in Fak mutant epidermal cells, in conjunction with the formation of abnormal focal adhesions distributed in different directions (Schober et al., 2007). Interestingly, although netrin 1 does not seem to affect axon length in cortical neurons (Dent et al., 2004), it increases the number of filopodia in hippocampal neurons (Lebrand et al., 2004; Shekarabi et al., 2005). In addition, interference with N-WASP activity using a dominant-negative form of this protein blocks the netrin 1-dependent increase in filopodia formation observed in spinal cord interneurons (Shekarabi et al., 2005). Thus, it seems plausible that loss of FAK function might alter different and/or convergent signaling cascades that regulate cytoskeletal and focal adhesion dynamics during the orchestration of growth cone motility and axon outgrowth. Our work indicates that FAK controls one of these signaling pathways by regulating actin nucleation through N-WASP phosphorylation.

\section{$\mathbf{N}$-WASP requires FAK to fulfill its functions in mediating filopodia formation}

Lamellipodia and filopodia in the growth cone display different dynamics of actin-filament assembly that can be stimulated de novo by actin nucleators. The spatiotemporal activity of these nucleators, which are typically auto-inhibited or intrinsically inactive in the cell, is tightly regulated. One of the main activators of these proteins is N-WASP, a protein associated with the formation of membrane protrusions (Pak et al., 2008). N-WASP interacts with CDC42 to modify the auto-inhibited conformation of the ARP2/3 complex, leading to its activation (Prehoda et al., 2000). In addition, phosphorylation of WASP promotes its translocation to filopodia in macrophages (Cory et al., 2002). Neurons lacking FAK accumulate less N-WASP protein at the growth cone, suggesting that FAK regulates N-WASP levels. The reduction in N-WASP clusters seems to be independent of the putative regulation of N-WASP transcription by FAK, as overexpression of $N$-WASP $P^{w t}-G f p$ in FAK-deficient neurons did not restore the density of N-WASP clusters to control levels, and NWASP protein levels are normal in cortical neurons lacking FAK. In addition, translocation or translation of N-WASP to the growth cone appears to be unaffected in FAK mutant neurons, as no change in the density of N-WASP clusters was observed in the axonal segment closest to the growth cone. A reasonable explanation for the decrease in N-WASP in the absence of FAK may be that FAK protects N-WASP from degradation through protein phosphorylation in the growth cone. Indeed, N-WASP phosphorylation precludes N-WASP proteasomal-dependent degradation (Park et al., 2005). Consistent with this idea, $N$ $W A S P^{Y 256 D}-G f p$ expression, but not that of $N-W A S P^{w t}-G f p$, returned the number of GFP-positive dots found in FAK mutant growth cones to wild-type levels (supplementary material Fig. S5).

What is the role of phosphorylated N-WASP in the growth cone? In macrophages, a constitutively phosphorylated form of WASP has been associated with actin polymerization in vitro and with filopodia formation (Cory et al., 2002). Accordingly, we found that the phosphorylated form of N-WASP accumulates in the peripheral region of the growth cone in neurons. Moreover, we demonstrated that expression of $N$-WASP $P^{256 D}-G f p$ but not $N$-WASP $P^{w t}-G f p$ restores the number of filopodia present in Fak mutant growth cones to control levels. Altogether, our results indicate that NWASP phosphorylation is required for FAK to promote filopodia formation through actin polymerization.

\section{Actin nucleation in filopodia functions through FAK and $\mathbf{N}$-WASP}

In the growth cone, lamellipodia are filled with a branched network of actin, whereas filopodia are packed with tight parallel bundles of filamentous actin (Mattila and Lappalainen, 2008). N-WASP has been linked with the activation of the ARP2/3 complex that is required for actin nucleation (Pollard and Borisy, 2003), although its function in the growth cone might be broader, depending on its 
location. A working model for filopodia formation proposes that ARP2/3 nucleates F-actin and forms branched actin superstructures typical of lamellipodia, which then align to form the bundles of Factin at the core of the filopodium (Svitkina et al., 2003; Vignjevic et al., 2003). It is tempting to speculate that N-WASP associates with ARP2/3 complexes independently of FAK to nucleate actin arrays in lamellipodia. Once this arrangement has been stabilized, FAK-mediated N-WASP phosphorylation might then promote its translocation to more peripheral regions and its association with other actin nucleators, such as formin, nucleating actin filaments and developing filopodia (supplementary material Fig. S7). A single protein (FAK) that orchestrates the formation of both branched and linear actin networks would increase the efficiency of the system in response to any rapid change. Indeed, studies in Drosophila have shown that the protein WASH (member of WASP/WAVE family protein) can control the formation of both types of actin structures through its interaction with ARP2/3 or Spire and formin Cappuccino (Liu et al., 2009). Further studies will be required to explore these connections in more depth.

\section{Acknowledgements}

We thank D. Baeza, G. Fernández and C. Serra for technical assistance; G Expósito and M. Calvo for assistance with the confocal microscopy; M. Maravall and C. Castellanos for statistic analyses; O. Marín, P. Fazzari, A. Nieto and S. Peregrin for critical reading of the manuscript; members of the Rico and Marín labs for valuable discussions and ideas; and M. Sefton for editorial assistance. We are grateful to H. E. Beggs and L. F. Reichardt, and to S. Goebbels and K. A. Nave for Fak floxfllox and NEX-Cre mice, respectively; and B. Sauer ( $p C S 2-C r e-E G f p), A$. Barrallo ( $p C S 2-m R F P)$, T. Parsons (FRNK, mycFakwt, mycFak ${ }^{Y 397 F}$ and mycFak ${ }^{K 454 R}$ ) and T. Takenawa (pCDL-SR-N-WASP) for plasmids.

\section{Funding}

This work was supported by grants from the Marie Curie International Reintegration Program of the European Commission through Research Project Contract SYNAPSON [MIRG-CT-2004-013082 to B.R.]; the Spanish Government [BFU2004-00349, SAF2007-61904, SAF2010-21723, to B.R. SAF2010-20604 to R.S.; BFU2010-17537 to M.M.; and CONSOLIDER CSD2007-00023 to B.R.]; and the following Foundations: Areces, Reina Sofía, Rioja Salud (to M.M.) and Alicia Koplowitz (to B.R.). B.R. is a European Molecular Biology Organization (EMBO) Young Investigator. Deposited in PMC for immediate release.

\section{Competing interests statement}

The authors declare no competing financial interests.

\section{Supplementary material}

Supplementary material available online at

http://dev.biologists.org/lookup/suppl/doi:10.1242/dev.080564/-/DC1

\section{References}

Barallobre, M. J., Del Río, J. A., Alcántara, S., Borrell, V., Aguado, F., Ruiz, M., Carmona, M. A., Martín, M., Fabre, M., Yuste, R. et al. (2000). Aberrant development of hippocampal circuits and altered neural activity in netrin 1deficient mice. Development 127, 4797-4810.

Bechara, A., Nawabi, H., Moret, F., Yaron, A., Weaver, E., Bozon, M., Abouzid, K., Guan, J. L., Tessier-Lavigne, M., Lemmon, V. et al. (2008). FAK-MAPK-dependent adhesion disassembly downstream of L1 contributes to semaphorin3A-induced collapse. EMBO J. 27, 1549-1562.

Beggs, H. E., Schahin-Reed, D., Zang, K., Goebbels, S., Nave, K.-A., Gorski, J., Jones, K. R., Sretavan, D. and Reichardt, L. F. (2003). FAK deficiency in cells contributing to the basal lamina results in cortical abnormalities resembling congenital muscular dystrophies. Neuron 40, 501-514

Chacón, M. R., Fernández, G. and Rico, B. (2010). Focal adhesion kinase functions downstream of Sema3A signaling during axonal remodeling. Mol. Cell. Neurosci. 44, 30-42

Challacombe, J. F., Snow, D. M. and Letourneau, P. C. (1996). Actin filament bundles are required for microtubule reorientation during growth cone turning to avoid an inhibitory guidance cue. J. Cell Sci. 109, 2031-2040.

Contestabile, A., Bonanomi, D., Burgaya, F., Girault, J. A. and Valtorta, F. (2003). Localization of focal adhesion kinase isoforms in cells of the central nervous system. Int. J. Dev. Neurosci. 21, 83-93.
Cory, G. O., Garg, R., Cramer, R. and Ridley, A. J. (2002). Phosphorylation of tyrosine 291 enhances the ability of WASp to stimulate actin polymerization and filopodium formation. Wiskott-Aldrich Syndrome protein. J. Biol. Chem. 277, 45115-45121.

Dent, E. W. and Kalil, K. (2001). Axon branching requires interactions between dynamic microtubules and actin filaments. J. Neurosci. 21, 9757-9769.

Dent, E. W., Barnes, A. M., Tang, F. and Kalil, K. (2004). Netrin-1 and semaphorin 3A promote or inhibit cortical axon branching, respectively, by reorganization of the cytoskeleton. J. Neurosci. 24, 3002-3012.

Dent, E. W., Kwiatkowski, A. V., Mebane, L. M., Philippar, U., Barzik, M., Rubinson, D. A., Gupton, S., Van Veen, J. E., Furman, C., Zhang, J. et al. (2007). Filopodia are required for cortical neurite initiation. Nat. Cell Biol. 9, 1347-1359.

Dickson, B. J. (2002). Molecular mechanisms of axon guidance. Science $\mathbf{2 9 8}$ 1959-1964.

Dwivedy, A., Gertler, F. B., Miller, J., Holt, C. E. and Lebrand, C. (2007), EnaNASP function in retinal axons is required for terminal arborization but not pathway navigation. Development 134, 2137-2146.

Fazzari, P., Paternain, A. V., Valiente, M., Pla, R., Luján, R., Lloyd, K., Lerma, J., Marín, O. and Rico, B. (2010). Control of cortical GABA circuitry development by Nrg1 and ErbB4 signalling. Nature 464, 1376-1380

Goebbels, S., Bormuth, I., Bode, U., Hermanson, O., Schwab, M. H. and Nave, K. A. (2006). Genetic targeting of principal neurons in neocortex and hippocampus of NEX-Cre mice. Genesis 44, 611-621.

Gupton, S. L. and Gertler, F. B. (2010). Integrin signaling switches the cytoskeletal and exocytic machinery that drives neuritogenesis. Dev. Cell 18, 725-736.

Katz, B. Z., Miyamoto, S., Teramoto, H., Zohar, M., Krylov, D., Vinson, C., Gutkind, J. S. and Yamada, K. M. (2002). Direct transmembrane clustering and cytoplasmic dimerization of focal adhesion kinase initiates its tyrosine phosphorylation. Biochim. Biophys. Acta 1592, 141-152.

Kwiatkowski, A. V., Rubinson, D. A., Dent, E. W., Edward van Veen, J., Leslie, J. D., Zhang, J., Mebane, L. M., Philippar, U., Pinheiro, E. M., Burds, A. A. et al. (2007). EnaNASP Is Required for neuritogenesis in the developing cortex. Neuron 56, 441-455.

Lebrand, C., Dent, E. W., Strasser, G. A., Lanier, L. M., Krause, M., Svitkina, T. M., Borisy, G. G. and Gertler, F. B. (2004). Critical role of EnaNASP proteins for filopodia formation in neurons and in function downstream of netrin-1. Neuron 42, 37-49.

Li, W., Lee, J., Vikis, H. G., Lee, S. H., Liu, G., Aurandt, J., Shen, T. L., Fearon, E. R., Guan, J. L., Han, M. et al. (2004). Activation of FAK and Src are receptor-proximal events required for netrin signaling. Nat. Neurosci. 7, 12131221

Lietha, D., Cai, X., Ceccarelli, D. F., Li, Y., Schaller, M. D. and Eck, M. J. (2007). Structural basis for the autoinhibition of focal adhesion kinase. Cell 129, 1177-1187.

Liu, G., Beggs, H., Jürgensen, C., Park, H. T., Tang, H., Gorski, J., Jones, K. R., Reichardt, L. F., Wu, J. and Rao, Y. (2004). Netrin requires focal adhesion kinase and Src family kinases for axon outgrowth and attraction. Nat. Neurosci. 7, 1222-1232

Liu, R., Abreu-Blanco, M. T., Barry, K. C., Linardopoulou, E. V., Osborn, G. E. and Parkhurst, S. M. (2009). Wash functions downstream of Rho and links linear and branched actin nucleation factors. Development 136, 2849-2860.

Martinez de Lagran, M., Benavides-Piccione, R., Ballesteros-Yañez, I., Calvo, M., Morales, M., Fillat, C., Defelipe, J., Ramakers, G. J. A. and Dierssen, M. (2012). Dyrk1A influences neuronal morphogenesis through regulation of cytoskeletal dynamics in mammalian cortical neurons. Cereb. Cortex. doi: 10.1093/cercor/bhr362.

Mattila, P. K. and Lappalainen, P. (2008). Filopodia: molecular architecture and cellular functions. Nat. Rev. Mol. Cell Biol. 9, 446-454.

Menegon, A., Burgaya, F., Baudot, P., Dunlap, D. D., Girault, J. A. and Valtorta, F. (1999). FAK+ and PYK2/CAKbeta, two related tyrosine kinases highly expressed in the central nervous system: similarities and differences in the expression pattern. Eur. J. Neurosci. 11, 3777-3788.

Miki, H., Sasaki, T., Takai, Y. and Takenawa, T. (1998). Induction of filopodium formation by a WASP-related actin-depolymerizing protein N-WASP. Nature 391, 93-96.

Morales, M., Colicos, M. A. and Goda, Y. (2000). Actin-dependent regulation of neurotransmitter release at central synapses. Neuron 27, 539-550.

Nawabi, H., Briançon-Marjollet, A., Clark, C., Sanyas, I., Takamatsu, H., Okuno, T., Kumanogoh, A., Bozon, M., Takeshima, K., Yoshida, Y. et al. (2010). A midline switch of receptor processing regulates commissural axon guidance in vertebrates. Genes Dev. 24, 396-410.

Owen, J. D., Ruest, P. J., Fry, D. W. and Hanks, S. K. (1999). Induced focal adhesion kinase (FAK) expression in FAK-null cells enhances cell spreading and migration requiring both auto- and activation loop phosphorylation sites and inhibits adhesion-dependent tyrosine phosphorylation of Pyk2. Mol. Cell. Biol. 19, 4806-4818.

Pak, C. W., Flynn, K. C. and Bamburg, J. R. (2008). Actin-binding proteins take the reins in growth cones. Nat. Rev. Neurosci. 9, 136-147. 
Park, S. J., Suetsugu, S. and Takenawa, T. (2005). Interaction of HSP90 to NWASP leads to activation and protection from proteasome-dependent degradation. EMBO J. 24, 1557-1570

Pollard, T. D. and Borisy, G. G. (2003). Cellular motility driven by assembly and disassembly of actin filaments. Cell 112, 453-465.

Prehoda, K. E., Scott, J. A., Mullins, R. D. and Lim, W. A. (2000). Integration of multiple signals through cooperative regulation of the N-WASP-Arp2/3 complex. Science 290, 801-806.

Price, C. J., Scott, R., Rusakov, D. A. and Capogna, M. (2008). GABA(B) receptor modulation of feedforward inhibition through hippocampa neurogliaform cells. J. Neurosci. 28, 6974-6982.

Rabut, G. and Ellenberg, J. (2004). Automatic real-time three-dimensional cell tracking by fluorescence microscopy. J. Microsc. 216, 131-137.

Ren, X. R., Ming, G. L., Xie, Y., Hong, Y., Sun, D. M., Zhao, Z. Q., Feng, Z., Wang, Q., Shim, S., Chen, Z. F. et al. (2004). Focal adhesion kinase in netrin-1 signaling. Nat. Neurosci. 7, 1204-1212.

Richardson, A. and Parsons, T. (1996). A mechanism for regulation of the adhesion-associated proteintyrosine kinase pp125FAK. Nature 380, 538-540.

Rico, B., Beggs, H. E., Schahin-Reed, D., Kimes, N., Schmidt, A. and Reichardt, L. F. (2004). Control of axonal branching and synapse formation by focal adhesion kinase. Nat. Neurosci. 7, 1059-1069.

Robles, E. and Gomez, T. M. (2006). Focal adhesion kinase signaling at sites of integrin-mediated adhesion controls axon pathfinding. Nat. Neurosci. 9, 1274 1283

Sánchez-Huertas C. and Rico, B. (2011). CREB-dependent regulation of GAD65 transcription by BDNF/TrkB in cortical interneurons. Cereb. Cortex 21, 777-788.

Schaller, M. D., Borgman, C. A., Cobb, B. S., Vines, R. R., Reynolds, A. B. and Parsons, J. T. (1992). pp125FAK a structurally distinctive protein-tyrosine kinase associated with focal adhesions. Proc. Natl. Acad. Sci. USA 89, 5192-5196.

Schaller, M. D., Hildebrand, J. D. and Parsons, J. T. (1999). Complex formation with focal adhesion kinase: A mechanism to regulate activity and subcellular localization of Src kinases. Mol. Biol. Cell 10, 3489-3505.

Schliwa, M. and van Blerkom, J. (1981). Structural interaction of cytoskeletal components. J. Cell Biol. 90, 222-235.

Schmitz, D., Frerking, M. and Nicoll, R. A. (2000). Synaptic activation of presynaptic kainate receptors on hippocampal mossy fiber synapses. Neuron 27, 327-338

Schober, M., Raghavan, S., Nikolova, M., Polak, L., Pasolli, H. A., Beggs, H. E., Reichardt, L. F. and Fuchs, E. (2007). Focal adhesion kinase modulates tension signaling to control actin and focal adhesion dynamics. J. Cell Biol. 176 667-680.

Serafini, T., Colamarino, S. A., Leonardo, E. D., Wang, H., Beddington, R., Skarnes, W. C. and Tessier-Lavigne, M. (1996). Netrin-1 is required for commissural axon guidance in the developing vertebrate nervous system. Cell 87, 1001-1014

Shekarabi, M., Moore, S. W., Tritsch, N. X., Morris, S. J., Bouchard, J. F. and Kennedy, T. E. (2005). Deleted in colorectal cancer binding netrin-1 mediates cell substrate adhesion and recruits Cdc42, Rac1, Pak1, and N-WASP into an intracellular signaling complex that promotes growth cone expansion. J. Neurosci. 25, 3132-3141.

Sprague, B. L. and McNally, J. G. (2005). FRAP analysis of binding: proper and fitting. Trends Cell Biol. 15, 84-91.

Star, E. N., Kwiatkowski, D. J. and Murthy, V. N. (2002). Rapid turnover of actin in dendritic spines and its regulation by activity. Nat. Neurosci. 5, 239-246.

Steketee, M. B. and Tosney, K. W. (2002). Three functionally distinct adhesions in filopodia: shaft adhesions control lamellar extension. J. Neurosci. 22, 80718083

Strasser, G. A., Rahim, N. A., VanderWaal, K. E., Gertler, F. B. and Lanier, L. M. (2004). Arp2/3 is a negative regulator of growth cone translocation. Neuron 43, 81-94

Suetsugu, S., Hattori, M., Miki, H., Tezuka, T., Yamamoto, T., Mikoshiba, K. and Takenawa, T. (2002). Sustained activation of N-WASP through phosphorylation is essential for neurite extension. Dev. Cell 3, 645-658.

Supèr, H. and Soriano, E. (1994). The organization of the embryonic and early postnatal murine hippocampus. II. Development of entorhinal, commissural, and septal connections studied with the lipophilic tracer Dil. J. Comp. Neurol. 344, 101-120.

Suter, D. M. and Forscher, P. (2000). Substrate-cytoskeletal coupling as a mechanism for the regulation of growth cone motility and guidance. $J$. Neurobiol. 44, 97-113.

Svitkina, T. M., Bulanova, E. A., Chaga, O. Y., Vignjevic, D. M., Kojima, S., Vasiliev, J. M. and Borisy, G. G. (2003). Mechanism of filopodia initiation by reorganization of a dendritic network. J. Cell Biol. 160, 409-421.

Vignjevic, D., Yarar, D., Welch, M. D., Peloquin, J., Svitkina, T. and Borisy, G. G. (2003). Formation of filopodia-like bundles in vitro from a dendritic network. J. Cell Biol. 160, 951-962.

Watanabe, F., Miyazaki, T., Takeuchi, T., Fukaya, M., Nomura, T., Noguchi, S., Mori, H., Sakimura, K., Watanabe, M. and Mishina, M. (2008). Effects of FAK ablation on cerebellar foliation, Bergmann glia positioning and climbing fiber territory on Purkinje cells. Eur. J. Neurosci. 27, 836-854.

Woo, S., Rowan, D. J. and Gomez, T. M. (2009). Retinotopic mapping requires focal adhesion kinase-mediated regulation of growth cone adhesion. $J$. Neurosci. 29, 13981-13991.

Wu, X., Suetsugu, S., Cooper, L. A., Takenawa, T. and Guan, J. L. (2004). Focal adhesion kinase regulation of N-WASP subcellular localization and function. J. Biol. Chem. 279, 9565-9576. 